\title{
Massive stars dying alone: the extremely remote environment of SN 2009ip
}

\author{
Nathan Smith, ${ }^{1 \star}$ Jennifer E. Andrews ${ }^{1}$ and Jon C. Mauerhan ${ }^{2}$ \\ ${ }^{1}$ Steward Observatory, University of Arizona, 933 N. Cherry Ave., Tucson, AZ 85721, USA \\ ${ }^{2}$ Department of Astronomy, University of California, Berkeley, CA 94720-3411, USA
}

Accepted 2016 August 29. Received 2016 August 23; in original form 2016 June 13

\begin{abstract}
We present late-time Hubble Space Telescope (HST) images of the site of supernova (SN) 2009ip taken almost $3 \mathrm{yr}$ after its bright 2012 luminosity peak. SN 2009ip is now slightly fainter in broad filters than the progenitor candidate detected by HST in 1999. The current source continues to be dominated by ongoing late-time circumstellar material interaction that produces strong $\mathrm{H} \alpha$ emission and a weak pseudo-continuum, as found previously for 1-2 yr after explosion. The intent of these observations was to search for evidence of recent star formation in the local ( $\sim 1 \mathrm{kpc} ; 10 \mathrm{arcsec})$ environment around SN 2009ip, in the remote outskirts of its host spiral galaxy NGC 7259. We can rule out the presence of any massive star-forming complexes like 30 Dor or the Carina nebula at the SN site or within a few kpc. If the progenitor of SN 2009ip was really a 50-80 $\mathrm{M}_{\odot}$ star as archival HST images suggested, then it is strange that there is no sign of this type of massive star formation anywhere in the vicinity. A possible explanation is that the progenitor was the product of a merger or binary mass transfer, rejuvenated after a lifetime that was much longer than 4-5 Myr, allowing its natal $\mathrm{H}$ II region to have faded. A smaller region like the Orion nebula would be an unresolved but easily detected point source. This is ruled out within $\sim 1.5 \mathrm{kpc}$ around SN 2009ip, but a small $\mathrm{H}_{\text {II }}$ region could be hiding in the glare of SN 2009ip itself. Later images after a few more years have passed are needed to confirm that the progenitor candidate is truly gone and to test for the possibility of a small $\mathrm{H}_{\text {II }}$ region or cluster at the $\mathrm{SN}$ position.
\end{abstract}

Key words: circumstellar matter-stars: evolution-supernovae: general-supernovae: individual: 2009ip - stars: winds, outflows.

\section{INTRODUCTION}

The class of Type IIn supernovae (SNe IIn hereafter), whose narrow $\mathrm{H}$ lines indicate strong interaction with dense circumstellar material (CSM), have challenged our understanding of stellar evolution and death. Their dense H-rich CSM, progenitor instability, and high initial masses inferred from various clues suggest a link to the class of luminous blue variables (LBVs), which are not supposed to be anywhere near core collapse in the standard scenario of massive single-star evolution (see Smith 2014 for a general review).

Among well-studied examples of SNe IIn, the explosion of SN 2009ip in mid-2012 (note that its discovery in 2009 was deemed to be a SN impostor) is one of the most interesting, with by far the best observational characterization of a directly detected progenitor among any SN in history (even SN 1987A). It had a (presumably) quiescent progenitor star candidate detected in archival $\mathrm{Hub}$ ble Space Telescope (HST) images, with a luminosity that implied a very high initial mass of at least 50-60 $\mathrm{M}_{\odot}$ (Smith et al. 2010; Foley et al. 2011). This source also showed a series of outbursts

\footnotetext{
^E-mail: nathans@as.arizona.edu
}

in the few years before the SN (Smith et al. 2010; Pastorello et al. 2013) that were reminiscent of both S Dor outbursts and giant eruptions of LBVs. Unlike any progenitor source so far, high-quality spectra of these precursor outbursts were obtained, with a detailed analysis before the $\mathrm{SN}$ indicating a strong similarity to LBVs (Smith et al. 2010; Foley et al. 2011). ${ }^{1}$ With a quiescent and very luminous progenitor, an S Dor outburst, several bright but brief SN impostor eruptions, and progenitor spectra resembling LBVs, SN 2009ip provides a strong link between LBVs and SNe IIn. In addition to the progenitor mass-loss rate requirements mentioned above, links to LBVs are also suggested by direct progenitor detections of other SNe IIn (Gal-Yam \& Leonard 2009; Smith et al. 2011), and by evidence of variable progenitor wind speeds in P Cygni absorption features of SNe IIn (Trundle et al. 2008).

The repeating variable source at the position of SN 2009ip began to brighten again in mid-2012, but this time things were different. Spectra of the fainter 2012a peak showed very broad P Cygni profiles with velocities of $13000 \mathrm{~km} \mathrm{~s}^{-1}$, suggesting that the

\footnotetext{
${ }^{1}$ Lower-quality, low-resolution photographic spectra were identified for SN 1987A (Walborn et al. 1989), but so far this is the only other SN with a progenitor spectrum.
} 
event was a core-collapse SN and not another LBV-like outburst (Smith \& Mauerhan 2012; Mauerhan et al. 2013). The subsequent and brighter 2012b event showed a high peak luminosity and a spectrum typical of SNe IIn with strong CSM interaction. The 2012 SN-like event has already been discussed extensively in the literature (Fraser et al. 2013, 2015; Mauerhan et al. 2013, 2014; Ofek et al. 2013; Pastorello et al. 2013; Prieto et al. 2013; Smith et al. 2013; Graham et al. 2014; Levesque et al. 2014; Margutti et al. 2014; Smith 2014; Martin et al. 2015; Moriya 2015).

In these publications and in discourse at meetings, there was some uncertainty and controversy about whether the 2012 event was a true core-collapse SN, since (1) CSM interaction can provide bright transients even from relatively low-energy explosions, (2) the initial SN (the 2012a event) was somewhat fainter than standard SNe II-P, and (3) the rich observational data set for the progenitor presented mysteries that were not easily explained by any existing model. Additional concerns were the apparently low ${ }^{56} \mathrm{Ni}$ mass implied by the data, and a lack of bright oxygen lines that are seen in the nebular phases of normal SNe II-P. Most of these questions are, however, related to the challenge in understanding $\mathrm{SNe}$ IIn and CSM interaction, rather than clear arguments against a corecollapse event. While it is difficult to prove definitively that the event was a core collapse because of the masking of CSM interaction, a core-collapse SN is the most straightforward explanation of the data. Smith, Mauerhan \& Prieto (2014) showed that all available evidence was consistent with the core-collapse SN explosion of a blue supergiant that encountered strong CSM interaction. In that picture, the 2012a event was faint because of the small stellar radius of the blue progenitor, and the implied ${ }^{56} \mathrm{Ni}$ mass is not so low (0.04 $\mathrm{M}_{\odot}$; roughly half of the value for SN 1987A) because the explosion date is at the start of the 2012a event. The influence of CSM interaction also changes the spectrum, making it difficult to draw conclusions from comparison to nebular phase spectra of normal SNe II-P. Moreover, both line-profile evolution (Smith et al. 2014) and spectropolarimetry (Mauerhan et al. 2014) show that the CSM interaction was highly aspherical and probably disc-like, forcing the kinetic energy budget of the event to be $\sim 10^{51} \mathrm{erg}$. Levesque et al. (2014) also argued for a disc-like CSM based on narrow line ratios. Fraser et al. (2015) showed that the source at +2 yr was consistent with steady ongoing CSM interaction with no additional outbursts, adding further evidence in favour of a corecollapse event.

SN 2009ip provides our clearest example of pre-SN instability that leads to eruptive pre-SN mass-loss in the few years before explosions, which may be associated with the final nuclear burning sequences in the last years of a massive star's life (Quataert \& Shiode 2012; Smith \& Arnett 2014). Alternative non-terminal models involving binary mergers and accretion were also proposed for the 2012 event (Kashi, Soker \& Moskovitz 2013; Soker \& Kashi 2013), but these cannot supply the required $10^{51}$ erg of kinetic energy.

In this paper, we are mainly concerned with the host galaxy environment around SN 2009ip. A fundamental interesting mystery was that while progenitor detections pointed to a very massive unstable star, the location of SN 2009ip was in the remote outskirts of its spiral host, far away from obvious signs of recent star formation and young stellar populations (Smith et al. 2010; Foley et al. 2011; Mauerhan et al. 2013; Margutti et al. 2014). It was located about $5 \mathrm{kpc}$ from the centre of its relatively small host spiral galaxy NGC 7259. By extrapolating the apparent metallicity gradient measured in the inner $\sim 1.5 \mathrm{kpc}$ out to the $5 \mathrm{kpc}$ radius of SN 2009ip, Margutti et al. (2014) infer a mildly subsolar metallicity at the SN site of $0.4<Z / Z_{\odot}<0.9$. SN 2009ip's progenitor can therefore be compared with populations of massive stars observed in the Milky Way and Large Magellanic Cloud (LMC).

A relevant recent result is that despite their high luminosities and high inferred initial masses, LBVs in the Milky Way and LMC appear to be relatively isolated compared to expectations for their presumed role in stellar evolution. Smith \& Tombleson (2015) demonstrated that LBVs selectively avoid clusters of O-type stars, especially early O-types that are their presumed progenitors. More importantly, LBVs are more dispersed on the sky than Wolf-Rayet (WR) stars; this rules out the standard picture wherein LBVs are a transitional phase between massive O-type stars and WR stars. ${ }^{2}$ Instead, Smith \& Tombleson (2015) suggested that most LBVs may be the result of interacting binary evolution, getting rejuvenated by either mass transfer or mergers. This would make them stand out as anomalously young compared to their surrounding populations. In other words, they are evolved massive blue stragglers. They may become even more isolated upon receiving a kick from their companion star's SN, although it is not yet clear if a kick is required to explain their environments. Also relevant to this story is that $\mathrm{SNe}$ IIn in general appear to be less correlated with bright $\mathrm{H} \alpha$ in their host galaxies than other types of SNe (Anderson et al. 2012; Habergham et al. 2014). While this has been interpreted as signifying lower initial masses by those authors, that interpretation has been a topic of debate (Crowther 2013; Smith \& Tombleson 2015). It is worth noting that inferences from statistics are challenged by the fact that both 'LBVs' and 'SNe IIn' are phenomenological classes, each of which may contain diverse members across a range of stellar mass. As described below, SN 2009ip seems to follow a similar trend of not having bright $\mathrm{H} \alpha$ nearby, despite having a detection of a very massive and luminous progenitor star.

\section{OBSERVATIONS}

The site of SN 2009ip was imaged with HST in 1999 using the F606W filter on Wide Field Planetary Camera 2 (WFPC2). A candidate progenitor source was detected within the error circle of SN 2009ip. The observations were presented and analysed by Smith et al. (2010) and Foley et al. (2011). From these data, both investigations inferred the existence of a massive progenitor, with an implied initial mass (compared to single-star evolutionary tracks) of $50-80 \mathrm{M}_{\odot}$ or $>60 \mathrm{M}_{\odot}$, respectively. ${ }^{3}$

Independent of stellar evolution models, a minimum luminosity (i.e. no bolometric correction or local extinction correction) of $>10^{5.9} \mathrm{~L}_{\odot}$ (Smith et al. 2010) would imply that the quiescent progenitor would need to be at least $25 \mathrm{M}_{\odot}$ in 1999, assuming that it was not exceeding the classical Eddington limit at that time. It would have had a significantly higher initial mass due to stellar wind mass-loss during its life. The fact that it appeared to suffer an S Dor-like eruption after this - followed by more extreme SN impostor-like luminosity spikes in 2009-2011 - lends weight

\footnotetext{
2 This has spawned a curious debate. Humphreys et al. (2016) advocated the idea that if one subdivides the LBV sample in a particular way, then the single-star picture of classical LBVs may still hold for 3 of the 19 Magellanic Cloud stars in the original sample of Smith \& Tombleson (2015). However, Smith (2016) demonstrated that there is no statistical significance to this claim, and that Humphreys et al. (2016) interpreted the relative separations of LBVs and comparison populations incorrectly.

${ }^{3}$ As in Smith et al. (2010), we adopt a distance modulus of $m-M=31.55 \mathrm{mag}$, and a Galactic reddening and extinction of $E(B-V)$ $=0.019 \mathrm{mag}$ and $A_{R}=0.05 \mathrm{mag}$, respectively, for NGC 7259 .
} 
Table 1. New HST WFC-UVIS images of SN 2009ip, including ST magnitudes for SN 2009ip and background $3 \sigma$ upper limits for point sources.

\begin{tabular}{lccccc}
\hline Date & Filter & Exp.(s) & ST mag & $1 \sigma$ & $3 \sigma$ UL $(\mathrm{mag})$ \\
\hline 2015 May 25 & $F 275 W$ & 2900 & 22.53 & 0.05 & 25.0 \\
2015 May 23 & $F 555 W$ & 1650 & 21.91 & 0.01 & 26.8 \\
2015 May 25 & $F 657 N$ & 5911 & 18.97 & 0.003 & 25.2 \\
2015 May 23 & $F 814 W$ & 1100 & 22.91 & 0.01 & 27.0 \\
\hline
\end{tabular}

to the idea that the $H S T$ progenitor in 1999 may have been the quiescent star, although the implied mass remains uncertain. In any case, the range of initial mass of $50-80 \mathrm{M}_{\odot}$ quoted by Smith et al. (2010) was determined from assuming the two possibilities that it was either (1) already in an LBV outburst in 1999 (with a cooler temperature and hence no bolometric correction, yielding $50 \mathrm{M}_{\odot}$ ), or (2) that it was a quiescent LBV (and hence in a hotter state, with a bolometric correction applied, implying $\sim 80 \mathrm{M}_{\odot}$ ).

We obtained new images of the same location at almost $3 \mathrm{yr}$ after the 2012 SN event using several filters with the WFC3-UVIS camera. Observational parameters are summarized in Table 1, which includes ST mag photometry for SN 2009ip itself, as well as $3 \sigma$ upper limits for point sources estimated from the background noise in the images in the region around SN 2009ip. We used three broadband filters to sample the UV/optical continuum ( $F 275 \mathrm{~W}, F 555 \mathrm{~W}$, and $F 814 W$ ), plus $F 657 N$ to sample low-velocity $\mathrm{H} \alpha$ emission at the host redshift. The photometry corresponds to days 1033 and 1035 after the date of discovery of the 2012a burst, following Mauerhan et al. (2013). The data for each filter were corrected for charge transfer efficiency (CTE) trails using the software tools available through STScI ${ }^{4}$. Cosmic rays (CRs) were removed using a CR-split pair of images for $F 657 N$, and for the broad-band filters, we used the LA-COSMIC package (van Dokkum 2001) with suggested parameters. We note that in the original uncorrected images, there were no CRs at the position of SN 2009ip; the nearest CRs in each image were separated by a distance of 6 pixels $(F 275 W), 14$ pixels $(F 555 W)$, and 7 pixels $(F 814 W)$. The final CTE and CR corrected images were combined and corrected for distortion effects using AstroDrizzle ${ }^{5}$. We retained the original pixel scale in the drizzle process.

The extremely remote environment shown in the new HST images (Fig. 1) is surprising, as discussed below. To help interpret the lack of any diffuse $\mathrm{H} \alpha$ emission detected in the vicinity of SN 2009ip, we injected fake sources into the $F 657 \mathrm{~N}$ image to illustrate how bright various types of $\mathrm{H}$ II regions should be. We obtained flux-calibrated images of 30 Dor and the Carina nebula from the Southern H-Alpha Sky Survey Atlas (SHASSA; Gaustad et al. 2001) and scaled these as they would appear if they were located in the outer parts of NGC 7259 at a distance of $20.4 \mathrm{Mpc}$. The SHASSA image of the Orion nebula is badly saturated in the Huygens region around the Trapezium, so we used an image of the Orion nebula obtained with the WFI camera at the European Southern Observatory/MPIA $2.2 \mathrm{~m}$ telescope on the nights of 2005 January 1-2 (Da Rio et al. 2009), which was made available through the $H S T$ archive as a product from the Treasury project on Orion ${ }^{6}$.

We also obtained a late-time spectrum of the site of SN 2009ip using the Bluechannel spectrograph on the MMT on 2015 November 2, which corresponds to day 1196 after the date of discovery. This spectrum was obtained with the $1200 \mathrm{lpm}$ grating covering the

\footnotetext{
${ }^{4}$ http://www.stsci.edu/hst/wfc3/tools/cte_tools

${ }^{5}$ See http://www.stsci.edu/hst/HST_overview/drizzlepac

${ }^{6}$ https://archive.stsci.edu/prepds/orion/
}

wavelength range from $\mathrm{Na}$ I $\mathrm{D}$ to $\mathrm{H} \alpha$, and was reduced using standard techniques. This was the same as in our previous MMT spectra of SN 2009ip (Mauerhan et al. 2013, 2014; Smith et al. 2014). The spectrum shows strong intermediate-width $\left( \pm 1000 \mathrm{~km} \mathrm{~s}^{-1}\right) \mathrm{H} \alpha$ emission, but any continuum or other emission features are below the noise level. The purpose of this spectrum was mainly to estimate the $\mathrm{H} \alpha$ line strength at late times so that we could appropriately scale a deeper spectrum of an old SN IIn at a similar time after explosion (in this case we chose SN 2005ip; see below).

\section{RESULTS}

\subsection{SN 2009ip late-time photometry}

The new HST images show a clearly detected point source at the location of SN 2009ip. Based on the measured offset from a nearby bright star in the field, this source is coincident with the position of the 1999 progenitor to within 0.043 arcsec (roughly 10 per cent of a WFC3 pixel). The late-time source has a profile consistent with the point spread function (PSF) in all the broad filters. Fig. 4 shows the result of subtracting a model PSF from the $F 657 N$ image of SN 2009ip. The model PSF was generated using the TINYTIM $\operatorname{program}^{7}$ (Krist, Hook \& Stoehr 2011). There is residual emission after the PSF subtraction at a typical brightness level of $0.5 \times 10^{-15} \mathrm{erg} \mathrm{s}^{-1} \mathrm{~cm}^{-2} \operatorname{arcsec}^{-2}$ located within $0.4 \operatorname{arcsec}(40 \mathrm{pc})$ of the SN site. This could indicate some faint extended $\mathrm{H}$ II region emission from a fading star-forming region. However, there are negative residuals at a similar level, so these are also consistent with being simple subtraction artefacts due to an imperfect PSF match. There is no detected emission brighter than $\sim 2 \times 10^{-16} \mathrm{erg}$ $\mathrm{s}^{-1} \mathrm{~cm}^{-2} \operatorname{arcsec}^{-2}$ beyond $40 \mathrm{pc}$ from the SN site. We also tried using a PSF made from a bright star on the same image, with similar results. PSF subtraction of the broad-band images (not shown) yielded similarly inconclusive results. In the continuum images, the residuals allow for the presence of a faint $\mathrm{SN}$ light echo or extended and very faint $\mathrm{OB}$ association that is $2-5$ per cent as bright as the $\mathrm{SN}$ point source, but as with $F 657 N$, the residuals are also consistent with subtraction artefacts from an imperfect PSF match.

The $F 555 \mathrm{~W}$ source is slightly fainter than the $F 606 \mathrm{~W}$ progenitor, but consistent with no change in the continuum level within the uncertainty. This is especially true when we consider that the $F 606 \mathrm{~W}$ image of the progenitor included $\mathrm{H} \alpha$ emission, while the more recent $F 555 \mathrm{~W}$ image does not. In a preliminary report of groundbased photometry, Thoene et al. (2015) also reported that SN 2009ip continued to fade, and by a few months after our HST images were taken, it was reported to be fainter than the progenitor.

The faint emission detected in the broad filters could be dominated by continuum from an underlying star cluster, late-time CSM interaction emission from the fading SN itself, or a combination of these two. In fact, there must be some contribution from CSM interaction emission, due to the strong and broad $\mathrm{H} \alpha$ detected in the late-time spectrum (Fig. 3). Fraser et al. (2015) showed that there was still strong CSM interaction at around $2 \mathrm{yr}$ after explosion. We can therefore rule out the possibility that there is no change in the brightness of the progenitor candidate (as would be the case if it was a chance alignment with an unrelated star), and so the progenitor must have faded by whatever amount is now contributed by CSM interaction luminosity. The possibility that a progenitor survived but is now obscured by dust is hard to rule out because it requires

\footnotetext{
${ }^{7}$ http://tinytim.stsci.edu/cgi-bin/tinytimweb.cgi
} 

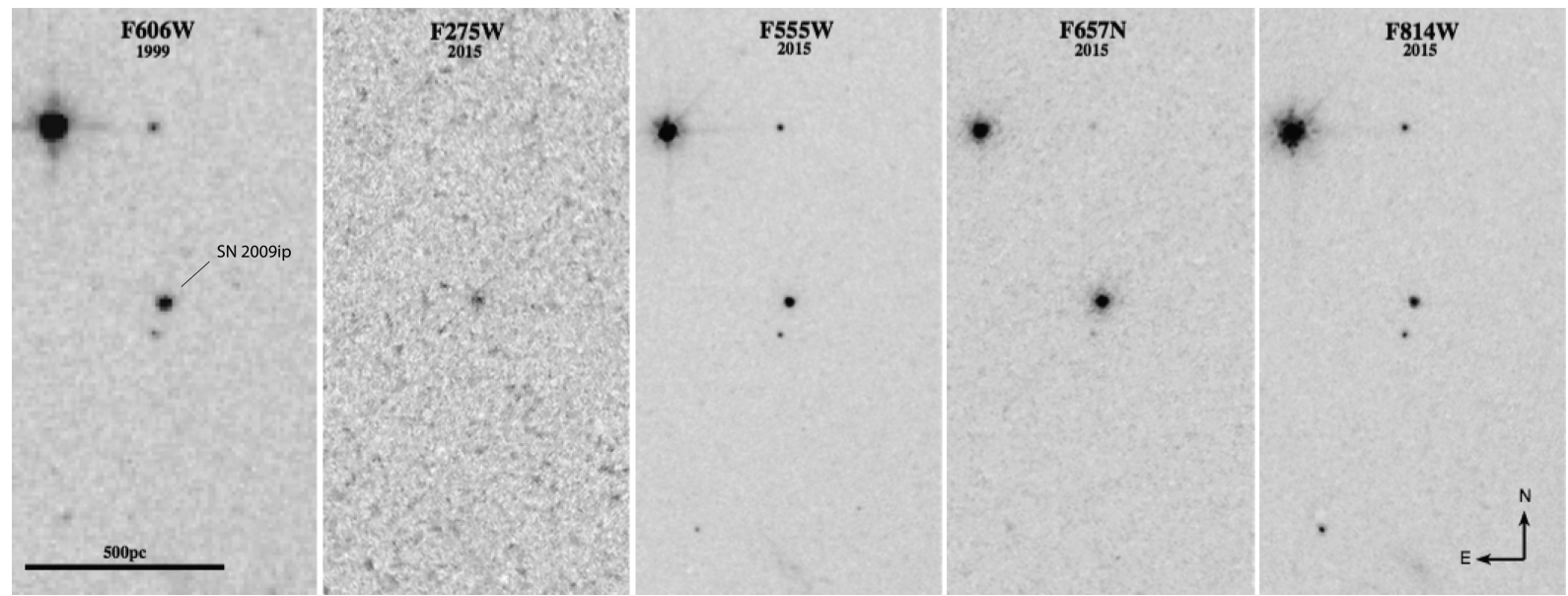

Figure 1. HST images of the immediate environment of SN 2009ip. The left-hand panel is the same WFPC $2 F 606 W$ image of the progenitor obtained in 1999 , from Smith et al. (2010). The other four panels are the new WFC3-UVIS images in F275W, F555W, F657N, and $F 814 W$ obtained in 2015 May (see Table 1). SN 2009ip is the brighter of the two sources at the centre of the image.

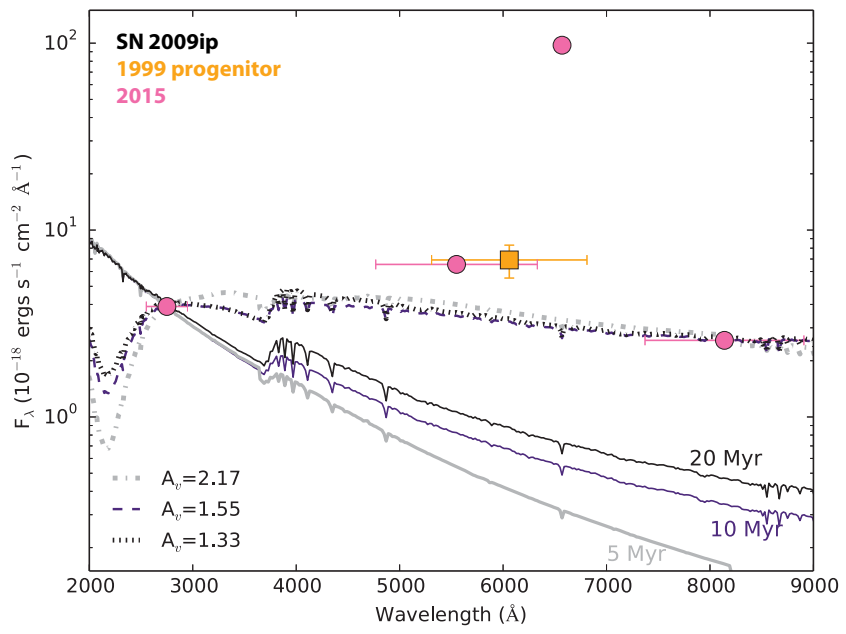

Figure 2. The photometry of the $F 606 \mathrm{~W}$ progenitor candidate in 1999 (orange square) and the photometry of the source detected in new HST images in 2015 (pink circles). These are compared to Starburst99 models as discussed in the text for 5, 10, and $20 \mathrm{Myr}$ (grey, blue, and black, respectively). Models with these ages and no reddening (solid) are scaled to the $F 275 \mathrm{~W}$ point, and the same models with reddening (dashed/dotted) are scaled to match the $F 275 W$ and $F 814 W$ points. See Table 2 and text.

some arbitrary amount of extinction to compensate for CSM interaction emission. However, Fraser et al. (2015) noted that there was no clear indication of new dust formation in the late-time data for SN 2009ip, so this would seem to argue that the progenitor candidate (Smith et al. 2010) has in fact faded.

Fig. 2 compares the observed spectral energy distribution in the HST photometry to the hypothetical emission from underlying young star clusters using examples from Starburst99 models (Leitherer et al. 1999). We show models with ages of 5, 10, and $20 \mathrm{Myr}$ scaled to the $F 275 \mathrm{~W}$ point only (assuming that the opti$\mathrm{cal} /$ red emission is dominated by the $\mathrm{SN}$ ), as well as these same cluster model spectra with reddening to match both the $F 275 \mathrm{~W}$ and the $F 814 W$ flux. Clusters much older than $20 \mathrm{Myr}$ would have turn-off masses too low (less than $12 \mathrm{M}_{\odot}$ ). The associated cluster terminal-age main-sequence masses ( $M_{\text {TAMS }}$ ), absolute $V$ mags, and
Table 2. Starburst 99 clusters matched to SN 2009ip photometry (see Fig. 2), with TAMS turn-off mass ( $\left.M_{\text {TAMS }}\right)$, absolute $V$ magnitude of the cluster $\left(M_{V}\right)$, and total cluster mass $\left(M_{\mathrm{cl}}\right) \cdot E(B-V)$ is the amount of reddening for the reddened models in Fig. 2.

\begin{tabular}{lcccc}
\hline $\begin{array}{l}\text { Age } \\
(\mathrm{Myr})\end{array}$ & $\begin{array}{c}M_{\text {TAMS }} \\
\left(\mathrm{M}_{\odot}\right)\end{array}$ & $\begin{array}{c}E(B-V) \\
(\mathrm{mag})\end{array}$ & $\begin{array}{c}M_{V} \\
(\mathrm{mag})\end{array}$ & $\begin{array}{c}M_{\mathrm{cl}} \\
\left(\mathrm{M}_{\odot}\right)\end{array}$ \\
\hline 5 & 54 & 0.00 & -6.99 & 15 \\
10 & 21 & 0.00 & -7.42 & 40 \\
20 & 12 & 0.00 & -7.65 & 100 \\
5 & 54 & 0.70 & -9.14 & 800 \\
10 & 21 & 0.50 & -9.02 & 710 \\
20 & 12 & 0.43 & -8.97 & 1120 \\
\hline
\end{tabular}

total cluster stellar masses are listed in Table 2. The corresponding absolute $V$ mag and total cluster mass corrected for extinction are also shown, along with the corresponding $E(B-V)$ value.

If we assume that CSM interaction dominates the optical/red emission, the unreddened clusters allowed by the $F 275 \mathrm{~W}$ photometry imply total cluster masses that are unreasonably low (lower than the progenitor mass of an individual star; this is because they have been artificially scaled to match the photometry). We discount these. Adding reddening to the host cluster, we can account for the $F 275 \mathrm{~W}$ and $F 814 W$ fluxes reasonably well, but there is an $F 555 W$ excess flux of about a factor of 2 above the model spectra, which could perhaps be accounted for by SN emission (see below). The stellar masses implied are more reasonable, but the problem with these reddened clusters is that the required reddening is larger than the value of the total $E(B-V)$ inferred for the line of sight of $E(B-V)$ $=0.019$ mag (Smith et al. 2010). A dominant contribution from a reddened cluster therefore seems unlikely as well. Overall, we find that an underlying star cluster does not provide a compelling explanation for the observed photometry, but we cannot rule out some contribution from a small cluster or association hidden by SN 2009ip. The total mass of any such cluster is constrained to be smaller than the total mass of the Orion nebula cluster (see below).

Another possibility is that the current photometry of SN 2009ip is dominated by late-time $\mathrm{SN}$ emission from ongoing CSM 


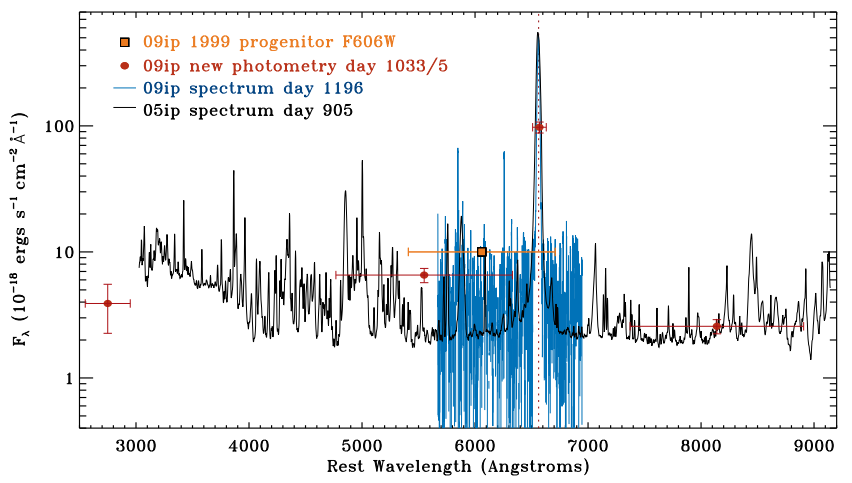

Figure 3. The new HST photometry from Table 1 compared to a roughly contemporaneous spectrum of SN 2009ip (blue) taken with the MMT in 2015 November, as well as a late-time day 905 spectrum of SN 2005ip (black) from Smith et al. (2009). SN 2005ip had stronger CSM interaction at almost 3 yr after explosion, and a spectrum with much higher signal-tonoise showing the fainter emission features that are expected to accompany strong $\mathrm{H} \alpha$ emission. This SN 2005ip spectrum has been scaled to match the same $\mathrm{H} \alpha$ flux as SN 2009ip, and can plausibly account for the 'continuum' emission in the broad filters.

interaction. Fig. 3 shows the SN 2009ip photometry compared to a spectrum that we obtained at the MMT several months later in 2015 November. This spectrum indicates that very strong $\mathrm{H} \alpha$ emission with a resolved width of about $2000 \mathrm{~km} \mathrm{~s}^{-1}$ continued to be observed, requiring the presence of strong ongoing CSM interaction at these late times. For comparison, Fig. 3 shows a late-time (day 905) spectrum of SN 2005ip from Smith et al. (2009). SN 2005ip was a Type IIn with very strong late-time CSM interaction, and this has much higher signal-to-noise across a wide wavelength range that demonstrates the lower level emission in many lines that may accompany bright $\mathrm{H} \alpha$. Many of these fainter emission lines blend together to make a blue 'pseudo-continuum' (Smith et al. 2009). The SN 2005ip spectrum has been scaled to match the same $\mathrm{H} \alpha$ flux as measured in the spectrum of SN 2009ip, providing a rough indication of the pseudo-continuum emission in HST filters that we might expect from SN 2009ip. We do not necessarily expect the late-time spectrum of SN 2009ip to be exactly the same as that of SN 2005ip. The point of this comparison is to illustrate that the level of CSM interaction we detect via the $\mathrm{H} \alpha$ emission in SN 2009ip should be accompanied by a host of other emission lines across a wide wavelength range that contribute to the broad-band filters. Using SN 2005ip as a guide, it seems possible that CSM interaction may account for most of the broad-band flux and relatively blue colour. A more detailed analysis of the late-time spectrum and its variability is postponed to a later paper.

Because the late-time CSM interaction emission must make some contribution to the observed $F 555 \mathrm{~W}$ photometry in 2015, we conclude that any underlying continuum source other than CSM interaction should be fainter than the $F 606 W$ progenitor seen in 1999 . Again, note that the $F 606 W$ image contains $\mathrm{H} \alpha$ whereas $F 555 W$ does not. However, we also note that the progenitor's $\mathrm{H} \alpha$ equivalent width was a factor of at least 10 weaker than the late-time source, so this may not be a strong effect (see Smith et al. 2014). It is likely that we will need to wait several more years to confirm that this source has actually faded enough to place strong constraints on the progenitor. The main goal of our proposed HST observations was to investigate the environment around SN 2009ip, as discussed next.

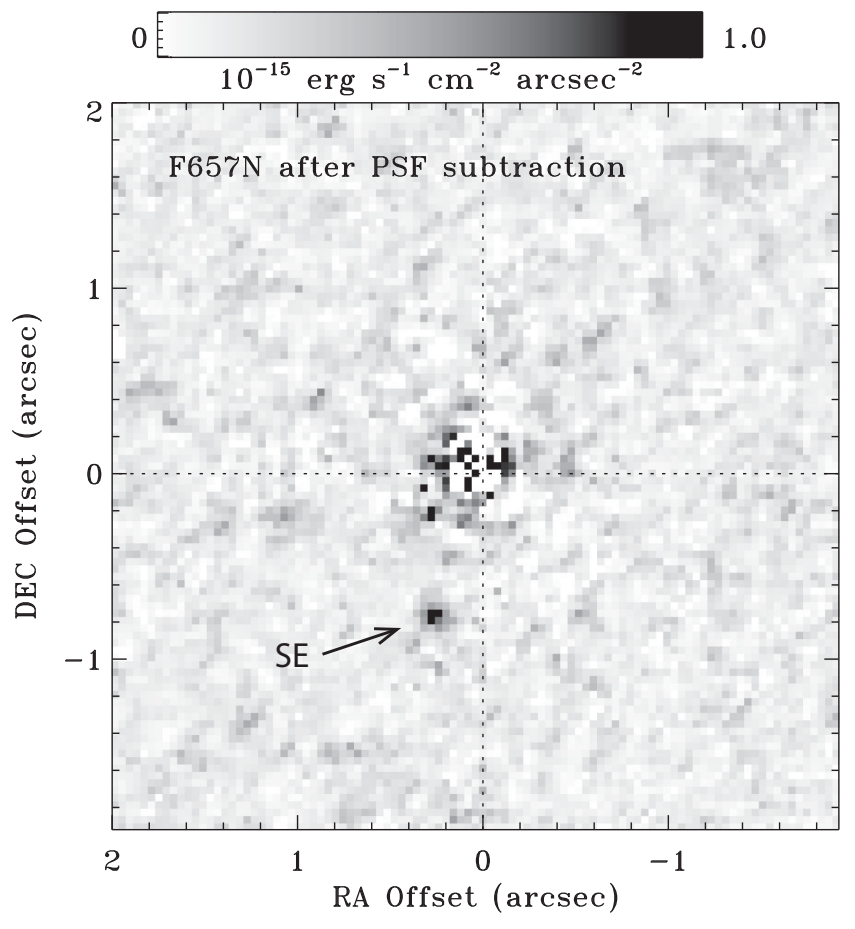

Figure 4. The new F657N image of the location of SN 2009ip after subtraction of a model PSF made with TINYTIM. There is residual extended emission at a level of roughly $0.5 \times 10^{-15} \mathrm{erg} \mathrm{s}^{-1} \mathrm{~cm}^{-2} \operatorname{arcsec}^{-2}$, but there are negative residuals at a similar level, so these could be subtraction artefacts from an imperfect PSF match. There is no extended emission beyond 0.4 arcsec (40 pc) from the SN site brighter than $\sim 0.2 \times 10^{-15} \mathrm{erg} \mathrm{s}^{-1} \mathrm{~cm}^{-2} \operatorname{arcsec}^{-2}$. The source labelled 'SE' is discussed in Section 3.2.

\subsection{A remote environment within $1 \mathrm{kpc}$}

The comparison between photometry of SN 2009ip and Starburst99 model spectra in Fig. 2 shows that our broad-band images could easily detect any massive young clusters or $\mathrm{OB}$ associations in these images. The sensitivity determined from $3 \sigma$ upper limits in the background of these images indicates that we would have detected sources with $M_{V}$ of $-4.7 \mathrm{mag}$, almost 100 times fainter than the SN 2009ip point source. This limit corresponds to a single mid to late O-type main-sequence star, or a single red supergiant. The $F 275 \mathrm{~W}$ image rules out even relatively small clusters with ages younger than $10 \mathrm{Myr}$ or any early O-type main-sequence stars (initial masses above $40 \mathrm{M}_{\odot}$ ); these would be easily detected in the $\sim 1 \mathrm{kpc}$ environment around SN 2009ip. No such bright clusters or luminous stars are seen. We take this as indicating a remarkable lack of recent massive star formation in the vicinity over the past $\sim 10 \mathrm{Myr}$.

In the vicinity of SN 2009ip, there is only one relatively faint point source about $100 \mathrm{pc}$ in projection to the south-east (labelled 'SE' in Figs 4 and 5). This source is, however, more than 10 times fainter than SN 2009ip at most wavelengths (except $F 814 W$, where it is five times fainter), redder, and not detected in $F 275 W$. Magnitudes for source $\mathrm{SE}$ are given in Table 3. The source is almost 100 times fainter than SN 2009ip in the $F 657 N$ filter, and the $F 657 N$ magnitude is consistent with continuum emission plus only a small contribution from $\mathrm{H} \alpha$ line emission (as much as half the flux in the narrow filter). Compared to Fig. 2 (where a cluster allowed by the much brighter source SN 2009ip was already quite small and/or old), any cluster associated with this SE source would need to be older than $20 \mathrm{Myr}$, would need to have very low total stellar mass and very 

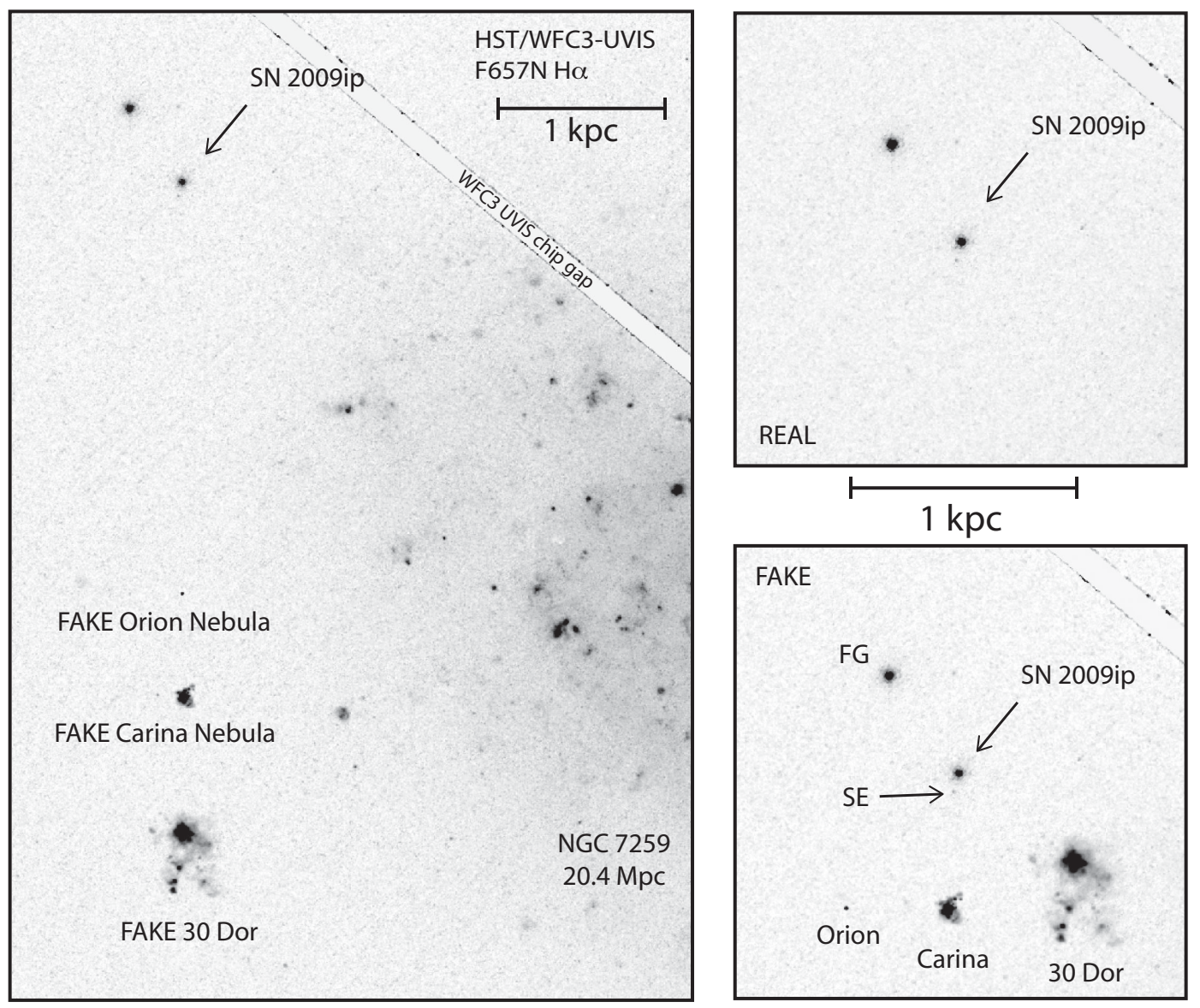

Figure 5. HST/WFC3-UVIS images of SN 2009ip's environment in the $F 657 N$ filter. The left-hand panel shows a wider view of the eastern half of the host galaxy, NGC 7259. In the lower-left corner, we have added in fake emission (made from real $\mathrm{H} \alpha$ images scaled to the appropriate distance) demonstrating what the Orion nebula, the Carina nebula, and the 30 Doradus complex would look like if they were located in the outer portions of NGC 7259 and observed with the sensitivity of our image. The two right-hand panels show a zoomed-in view of a 2 kpc box centred on SN 2009ip, with the real data (top) and a version with the same fake emission from Orion, Carina, and 30 Dor added in for comparison. The local environment of SN 2009ip shows essentially no evidence for recent massive star formation episodes in the form of young or ageing $\mathrm{H}$ II regions. 'FG' is a likely foreground star, and 'SE' is a nearby source discussed in Section 3.2.

Table 3. HST WFC-UVIS photometry of the point source SE to the south-east of SN 2009ip obtained in 2015.

\begin{tabular}{lccc}
\hline Filter & ST mag & $1 \sigma$ & Abs. mag \\
\hline$F 275 W$ & $>26.2$ & - & $>\lesssim 5.2$ \\
$F 555 W$ & 24.87 & 0.07 & $\lesssim 6.65$ \\
$F 657 N$ & 23.69 & 0.11 & $\lesssim 7.83$ \\
$F 814 W$ & 24.69 & 0.05 & $\lesssim 6.83$ \\
\hline
\end{tabular}

faint residual $\mathrm{H} \alpha$ nebulosity, and/or would need a large foreground extinction. If SN 2009ip were a runaway from this cluster, then it is a remarkably isolated and ageing star cluster. These considerations make it very unlikely that SE is a young birth cluster of SN 2009ip that could have spawned a $>50 \mathrm{M}_{\odot}$ single star, but perhaps it may have spawned a $\sim 20 \mathrm{M}_{\odot}$ in the rejuvenated binary scenario discussed below. It seems more likely, however, that SE is simply a nearby supergiant (with an initial mass of perhaps $10-20 \mathrm{M}_{\odot}$ based on its luminosity) with some $\mathrm{H} \alpha$ emission associated with a wind or nebula, or an older open cluster.

Extended $\mathrm{H} \alpha$ emission also serves as a tracer of any recent or ongoing massive star formation in the neighbourhood. Our $F 657 \mathrm{~N}$ image was designed to be sensitive to even relatively modest $\mathrm{H}$ II regions. Indeed, the $F 657 N$ image reveals dozens of $\mathrm{H}$ II regions with a wide range of surface brightness in the interior few $\mathrm{kpc}$ of the host galaxy nucleus, but there is a marked absence of any $\mathrm{H}_{\text {II }}$ emission in the outer regions of the galaxy beyond 3-4 kpc from the centre.

The new F657N image of SN 2009ip and its surroundings is shown in Fig. 5. This image reveals no extended $\mathrm{H} \alpha$ emission from any young $\mathrm{H}_{\mathrm{II}}$ regions in the surrounding $\sim 1 \mathrm{kpc}$ around SN 2009ip, which is found about $5 \mathrm{kpc}$ from the galaxy centre. There is a bright point source to the north-east (possibly a foreground star; ' $F G$ ' in Fig. 5) and two very faint point sources as well (including SE, which, as noted above, cannot be a young region), but all these other sources have similar brightness in broad-band continuum filters and so these are unlikely to be young $\mathrm{H}_{\text {II }}$ regions. SN 2009ip itself is much brighter in the $F 657 N$ filter, consistent with its bright $\mathrm{H} \alpha$ emission detected in the spectrum (Fig. 3).

To illustrate how the lack of $\mathrm{H} \alpha$ detection indicates a dearth of recent or ongoing massive star formation in the vicinity, Fig. 5 shows two panels that zoom in on the surrounding $\sim 1 \mathrm{kpc}$ around SN 2009ip. One of these is the observed F657N image, and the other is the same image with some fake comparison sources injected. As noted earlier in Section 2, we used $\mathrm{H} \alpha$ images of the Orion nebula, the Carina nebula, and the 30 Dor complex, and scaled them to 
the appropriate pixel size as they would appear in these images if they resided at the distance of NGC 7259. These sources were then convolved to the full width at half-maximum of the $F 657 N$ images and added to the FITS files of the HST data at the appropriate surface brightness for the flux calibration in the HST images. We also added these fake images to the left side of the larger scale image in the same figure. All three nebulae would have been easily detected in the images, although the Orion nebula is so small that it would have been an unresolved point source (note, however, that the Orion nebula is also found among a larger complex of star formation that we did not include this in the fake injected image).

It is evident that the eastern side of NGC 7259 has no extreme mini-starbursts comparable to 30 Dor, although there are a few detected $\mathrm{H} \alpha$ sources that have a scale in between 30 Dor and Carina, and several comparable to Carina in the inner spiral arms of the galaxy. There are a few dozen smaller $\mathrm{H}$ in regions comparable to Orion scattered around NGC 7259 , but none in the vicinity of SN 2009ip.

With a presumed initial mass of $50-80 \mathrm{M}_{\odot}$ (Smith et al. 2010; Foley et al. 2011), and a lifetime of only 3-4 Myr, one would expect SN 2009ip to reside in a massive young cluster with many other O-type stars, and to be surrounded by bright extended $\mathrm{H}$ il region emission. This is clearly not the case, nor are there any bright $\mathrm{H}$ II regions in the vicinity. We did not attempt to obtain deep radio data to search for free-free emission from $\mathrm{H}$ il regions or $\mathrm{SN}$ remnants, although this may be an interesting future observation to make. We discuss implications corresponding to each type of region below.

30 Dor: Big $\mathrm{H}$ II region complexes like 30 Dor can have ongoing massive star formation for 10-20 Myr (Crowther 2013). 30 Dor contains the very young (1-2 Myr) cluster R136, as well as the $\sim 10$ Myr old population that gave rise to SN 1987A. The total stellar mass is as much as $\sim 10^{5} \mathrm{M}_{\odot}$, more or less depending on how much of the population one includes around the R136 cluster. If SN 2009ip were in such a region, the new HST images would clearly detect bright extended $\mathrm{H} \alpha$ emission in the few $10^{2}$ pc surrounding SN 2009ip. We can rule out the presence of such a large complex at the position of SN 2009ip or within several kpc.

Carina: The carina nebula has birthed about 70 O-type stars (Smith 2006), and is of sufficient scale to sample the upper part of the main sequence. There are a handful of extremely massive stars in Carina, including $\eta$ Carinae and three very massive WNH stars. The total stellar mass is estimated to be roughly $5 \times 10^{4} \mathrm{M}_{\odot}$ (Smith \& Brooks 2007). Moderately large $\mathrm{H}_{\text {II }}$ regions like Carina fade in 4-5 Myr (Carina is now $\sim 3$ Myr old) when the most massive $\mathrm{O}$ stars have died and the bulk of the ionizing photons shut off. With a presumed initial mass of $50-80 \mathrm{M}_{\odot}$, one would expect $\mathrm{SN}$ 2009ip to come from a region that is no older than this (if it is a single star). However, we can clearly rule out a bright extended $\mathrm{H} \alpha$ nebula like Carina at the position of SN 2009ip (see Fig. 4). After about $5 \mathrm{Myr}$, there may be a larger and fainter $\mathrm{H} \alpha$ bubble. This is harder to rule out, and depends on an adopted escape fraction of ionizing photons. As noted earlier, our PSF subtraction residuals allow for some faint emission within $40 \mathrm{pc}$ of the SN site, but these could also be artefacts. The lack of bright extended emission to a surface brightness about 10 times fainter than Carina's outer shells (Smith \& Brooks 2007) would imply a region significantly older than 5 or $6 \mathrm{Myr}$, and would also therefore cause us to expect that any stars with initial mass $\geq 40 \mathrm{M}_{\odot}$ are long since dead. The lack of any Carina-like H II region in the $1 \mathrm{kpc}$ surrounding SN 2009ip also suggests that it is unlikely to be a runaway from such a region.

Orion: The Orion nebula contains only one O-type star (with an initial mass around $30 \mathrm{M}_{\odot}$ ), and a total stellar mass in the Orion nebula cluster of about $1800 \mathrm{M}_{\odot}$ (Hillenbrand \& Hartmann 1998).
Smaller $\mathrm{H}_{\text {II }}$ regions like this with only a couple $\mathrm{O}$ stars die after 5-6 Myr as well, when any mid-O-type stars evolve off the main sequence and most of the ionizing photons shut off. The surrounding gas shells become neutral and get much fainter and diffuse as they age. Moreover, even when young and bright, such a region would be very small in terms of angular extent and would be unresolved by $H S T$ at $20 \mathrm{Mpc}$. Such an $\mathrm{H}$ II region could easily be the birth environment of SN 2009ip and may be hiding under the $\mathrm{SN}$ emission. It would be unusual (although perhaps not impossible with stochastic sampling of the initial mass function; Andrews et al. 2014) to find such a massive $50-80 \mathrm{M}_{\odot}$ progenitor being born in this type of region. It seems more plausible that such an $\mathrm{H}$ II region might give rise to a very massive star like the progenitor of SN 2009ip through binary evolution (see below). Although an Orion-like region could be hiding underneath the current photometry of SN 2009ip, it is important to note that such regions are rarely found in complete isolation. Massive star formation tends to propagate from one region of a molecular cloud complex to another (Elmegreen \& Lada 1977), leading to chains and complexes of $\mathrm{H}$ II region emission in disc galaxies. Orion is part of a larger complex of star formation in the Orion region, but no sign of any other nearby $\mathrm{H}$ II regions are seen in the images of SN 2009ip. Therefore, we have the additional unusual circumstance that SN 2009ip's birth environment would need to be a singular instance of star formation, or the last such episode of massive star formation in the vicinity.

All of these considerations point to ages that are longer than $5 \mathrm{Myr}$, and more likely around $10 \mathrm{Myr}$ or more, suggesting initial masses around $20 \mathrm{M}_{\odot}$. This is in apparent contradiction with the high luminosity of the progenitor star in its early phase before the series of bright pre-SN eruptive events (at least $50-60 \mathrm{M}_{\odot}$ ).

One way to close the gap between the massive progenitor that was detected and the surprising lack of recent massive star formation in the vicinity would be to consider binary evolution. Either SN 2009ip was a runaway star born at some other location or it has been rejuvenated by binary interaction.

If it is a runaway, the nearest indications of massive star formation in NGC 7259 are found at least $1.5 \mathrm{kpc}$ away. To cover this distance in a time of $\sim 3 \mathrm{Myr}$ would require a kick speed of $400-500 \mathrm{~km} \mathrm{~s}^{-1}$. This would be a very fast kick for a massive companion of a $\mathrm{SN}$. Moreover, such high runaway speeds seem hard to reconcile with the narrow emission lines in the spectrum of SN 2009ip (arising in the pre-shock CSM), whose centroid radial velocities are consistent to within $\lesssim 100 \mathrm{~km} \mathrm{~s}^{-1}$ with the redshift of the host galaxy (Mauerhan et al. 2013).

A more likely alternative may be that the progenitor of SN 2009ip increased its mass and luminosity late in life by accreting mass in a mass transferring interacting binary system, or by a stellar merger event (see Paczynski 1971; Vanbeveren, De Loore \& Van Rensbergen 1998; Podsiadlowski et al. 2010; Langer 2012; Vanbeveren et al. 2013; Justham, Podsiadlowski \& Vink 2014). This would allow the stars to live a longer core H-burning main-sequence lifetime of a lower mass star, and to then get rejuvenated by mass accretion or merging to produce the luminous progenitor source that was detected in 1999. This is the sort of evolutionary scenario suggested for LBVs based on their remote environments (Smith \& Tombleson 2015), although it should be noted that SN 2009ip's environment seems unusually remote even among known LBVs. The most isolated LBV known in nearby galaxies is R71 in the LMC, which is $300 \mathrm{pc}$ from any O-type stars, but still on the outskirts of 30 Dor (Smith \& Tombleson 2015). Smith \& Tombleson (2015) pointed out, however, that rejuvenation by mass accretion or merging might produce an LBV-like star that appears very isolated even without a kick if it is a blue straggler in a relatively small, isolated, and 
ageing star cluster. This scenario requires the presence of a relatively modest $\sim 10 \mathrm{Myr}$ old star cluster or association at the position of SN 2009ip, which could be hidden by the current ongoing CSM interaction. Observations after the CSM interaction luminosity fades (this may take several years) can eventually test this hypothesis.

A binary scenario for the progenitor of SN 2009ip was invoked previously, independent of the remote environment. The disc-like CSM inferred to exist around SN 2009ip (Levesque et al. 2014; Mauerhan et al. 2014; Smith et al. 2014) would be consistent with the notion that some strong binary interaction had occurred in the recent past. The repeating brief luminosity spikes in 2009-2011 were also attributed to binary close encounters (Mauerhan et al. 2013, 2014; Smith 2014), either through direct stellar collisions at periastron in an eccentric system as in the $\eta$ Car system (Smith 2011) or potentially due to periastron accretion events (Kashi et al. 2013). The remote environment discussed here strengthens the argument for a binary progenitor. If the sort of binary rejuvenation discussed above is appropriate for SN 2009ip, then it is important to remember that the companion should still be there if the binary system did not merge. This companion might be combined with any host cluster light in the late-time photometry after the CSM interaction luminosity fades.

If this sort of binary rejuvenation scenario is not the origin of SN 2009ip, then its very luminous progenitor source is difficult to understand. To produce such a luminous, H-rich progenitor with a strong wind and eruptive variability may require a more exotic scenario involving a binary system with a massive compact object accreting from a companion, for example, which might then lead to a core-collapse $\mathrm{SN}$ explosion.

A pulsational pair instability (PPI) eruption (Woosley, Blinnikov $\&$ Heger 2007) is the only proposed single-star mechanism to produce a non-terminal eruption that could account for the total energetics of SN 2009ip's 2012 event, which has been shown to require a kinetic energy well above $10^{50}$ erg because of the asymmetric CSM and the large ejecta mass implied by the sustained broadlined spectrum (Mauerhan et al. 2013, 2014; Smith et al. 2014). However, typically the PPI will have a large ejecta mass and expansion speeds $\lesssim 5000 \mathrm{~km} \mathrm{~s}^{-1}$ (Woosley et al. 2007), slower than the observed $13000 \mathrm{~km} \mathrm{~s}^{-1}$ broad lines in SN 2009ip's 2012a event (Mauerhan et al. 2013). The most energetic PPI events approaching $10^{51}$ erg would require extremely high initial masses in excess of $100 \mathrm{M}_{\odot}$, and it is uncertain if they occur at all at $0.5 \mathrm{Z}_{\odot}$ due to mass-loss. It seems very unlikely that there would be no evidence whatsoever of recent massive star formation in the vicinity of SN 2009ip if it originated from a star born with such a high initial mass.

\section{ACKNOWLEDGEMENTS}

Support was provided by the National Aeronautics and Space Administration (NASA) through HST grant GO-13787 from the Space Telescope Science Institute, which is operated by AURA, Inc., under NASA contract NAS5-26555. Support was also provided by the National Science Foundation (NSF) through grants AST-1210599 and AST-1312221 to the University of Arizona. This study used data products from the Southern H-Alpha Sky Survey Atlas (SHASSA), which is supported by the National Science Foundation.
Andrews J. E. et al., 2014, ApJ, 793, 4

Crowther P. A., 2013, MNRAS, 428, 1927

Da Rio N., Robberto M., Soderblom D. R., Panagia N., Hillenbrand L. A., Palla F., Stassun K., 2009, ApJS, 183, 261

Elmegreen B. G., Lada C. J., 1977, ApJ, 214, 725

Foley R. J., Berger E., Fox O., Levesque E. M., Challis P. J., Ivans I. I., Rhoads J. E., Soderberg A. M., 2011, ApJ, 732, 32

Fraser M. et al., 2013, MNRAS, 433, 1312

Fraser M. et al., 2015, MNRAS, 453, 3886

Gal-Yam A., Leonard C. D., 2009, Nature, 458, 865

Gaustad J. E., McCullough P. R., Rosing W., Van Buren D., 2001, PASP, 113,1326

Graham M. L. et al., 2014, ApJ, 787, 163

Habergham S. M., Anderson J. P., James P. A., Lyman J. D., 2014, MNRAS, 441,2230

Hillenbrand L. A., Hartmann L. W., 1998, ApJ, 492, 540

Humphreys R. M., Weis K., Davidson K., Gordon M. S., 2016, ApJ, 825, 64

Justham S., Podsiadlowski P., Vink J. S., 2014, ApJ, 796, 121

Kashi A., Soker N., Moskovitz N., 2013, MNRAS, 436, 2484

Krist J. E., Hook R. N., Stoehr F., 2011, Proc. SPIE, 8127, 81270J

Langer N., 2012, ARA\&A, 50, 107

Leitherer C. et al., 1999, ApJS, 123, 3

Levesque E., Stringfellow G. S., Ginsburg A. G., Bally J., Keeney B. A., 2014, AJ, 147, 23

Margutti R. et al., 2014, ApJ, 780, 21

Martin J. C., Hambsch F. J., Margutti R., Tan T. G., Curtis I., Soderberg A., 2015, AJ, 149, 9

Mauerhan J. C. et al., 2013, MNRAS, 430, 1801

Mauerhan J. C. et al., 2014, MNRAS, 442, 1166

Moriya T. J., 2015, ApJ, 803, L26

Ofek E. O., Lin L., Kouveliotou C., Younes G., Göğüş E., Kasliwal M. M., Cao Y., 2013, ApJ, 768, 47

Paczynski B., 1971, ARA\&A, 9, 183

Pastorello A. et al., 2013, ApJ, 767, 1

Podsiadlowski P., Ivanova N., Justham S., Rappaport S., 2010, MNRAS, 406, 840

Prieto J. L., Brimacombe J., Drake A. J., Howerton S., 2013, ApJ, 763, L27

Quataert E., Shiode J., 2012, MNRAS, 423, L92

Smith N., 2006, MNRAS, 367, 763

Smith N., 2011, MNRAS, 415, 2020

Smith N., 2014, ARA\&A, 52, 487

Smith N., 2016, MNRAS, 461, 3353

Smith N., Arnett D., 2014, ApJ, 785, 82

Smith N., Brooks K. J., 2007, MNRAS, 379, 1279

Smith N., Mauerhan J. C., 2012, Astron. Telegram, 4412, 1

Smith N., Tombleson R., 2015, MNRAS, 447, 602

Smith N. et al., 2009, ApJ, 695, 1334

Smith N. et al., 2010, AJ, 139, 1451

Smith N. et al., 2011, ApJ, 732, 63

Smith N., Mauerhan J. C., Kasliwal M., Burgasser A., 2013, MNRAS, 434, 2721

Smith N., Mauerhan J. C., Prieto J. L., 2014, MNRAS, 434, 2721

Soker N., Kashi A., 2013, ApJ, 764, L6

Thoene C., de Ugarte Postigo A., Leloudas G., Cano Z., Maeda K., 2015, Astron. Telegram, 8417, 1

Trundle C., Kotak R., Vink J. S., Meikle W. P. S., 2008, A\&A, 483, L47

van Dokkum P. G., 2001, PASP, 113, 1420

Vanbeveren D., De Loore C., Van Rensbergen W., 1998, A\&AR, 9, 63

Vanbeveren D., Mennekens N., Van Rensbergen W., De Loore C., 2013, A\&A, 552, A105

Walborn N. R. et al., 1989, A\&A, 219, 229

Woosley S. E., Blinnikov S., Heger A., 2007, Nature, 450, 390

\section{REFERENCES}

Anderson J. P., Habergham S. M., James P. A., Hamuy M., 2012, MNRAS, 424,1372

This paper has been typeset from a $\mathrm{T}_{\mathrm{E}} \mathrm{X} / \mathrm{LAT} \mathrm{E} \mathrm{X}$ file prepared by the author. 